\title{
ЗАДАЧА ОПТИМАЛЬНОГО УПРАВЛЕНИЯ УРОВНЕМ ВЗРЫВООПАСНОСТИ ПОТЕНЦИАЛЬНО ВЗРЫВООПАСНОГО ОБЪЕКТА
}

\author{
Волков В.Э. ${ }^{1}$, Лобода Ю.Г. ${ }^{2}$, Макоед Н.А. ${ }^{3}$ \\ ${ }^{1}$ Одесский национальный университет им. И.И. Мечникова, Одесса \\ ${ }^{2,3} \mathrm{Oдесская} \mathrm{национальная} \mathrm{академия} \mathrm{пищевых} \mathrm{технологий,} \mathrm{Одесса}$ \\ ORCID: ${ }^{1} 0000-0002-3990-8126,{ }^{2} 0000-0003-4857-7389,{ }^{3} 0000-0003-4591-555 X$ \\ E-mail: ${ }^{1}$ viktor@te.net.ua, ${ }^{2}$ jul.loboda@gmail.com, ${ }^{3}$ natamakoyed@gmail.com
}

Copyright (C) 2018 by author and the journal "Automation technologies and business - processes. This work is licensed under the Creative Commons Attribution International License (CC BY). http://creativecommons.org/licanses/by/4.0

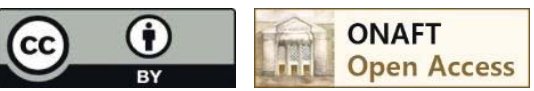

DOI: 10.15673/atbp.v10i1.874

Аннотация: Рассмотрен вопрос об оптимальном управлении уровнем взрьвоопасности потенциально взрывоопасного объекта. Задача поставлена математически в общем виде и решена для одного частного случая (в этом случае уровень взрывоопасности объекта полностью определяется соотношением размеров объекта и длинь преддетонационного участка). Решение основано на полученной ранее аналитической оценке длинь преддетонационного участка. Длина преддетонаџионного участка рассматривается как максимизируемая при определенных ограничениях функиия двух переменных. Указанные ограничения налагаются технологическим процессом и характером производства. Решение реализовано в программном продукте. Разработанная программа применяется для оптимального управления взрывобезопасностью отдельных силосов. Эта программа может стать важной составляющей программного обеспечения автоматизированной системы управления элеватором или другим зерновым предприятием, которое является потенциально взрывоопасным объектом. В результате расчетов пользователь (как правило, оператор-технолог) получает информацию о наиболее взрывобезопасном состоянии силоса из всех его возможных состояний. Эта информация является необходимой для принятия обоснованного решения по обеспечению взрывобезопасности силоса.

Abstract: Problem of the optimal control for the explosion hazard level of the potentially explosive object is considered. This problem is posed mathematically in a general form and solved for one particular case (in this case, the explosion hazard level of an object is completely determined by the ratio of sizes of this object and the length of the detonation induction distance). The solution is based on a previously obtained analytical estimate of the length of the detonation induction distance. The detonation induction distance is considered as $t$ he function of two variables to be maximized under certain restrictions. These restrictions are imposed by the technological process and nature of production. The solution is implemented in a software product. The designed program is used for optimal control of the explosion safety of individual silos. This program can become an important component of the software of an automated control system for an elevator or other grain enterprise, which is a potentially explosive object. As a result of the calculations, the user (as a rule, the process operator) receives information about the most explosion-proof state of the silo from all its possible states. This information is necessary to make a reasoned decision to ensure the safety of the silo.

Ключевые слова: оптимальное управление, взрыв, взрывоопасность, потенциально взрывоопасный объект, длина преддетонационного участка.

Keywords: optimal control, explosion, explosion hazard, potentially explosive object, detonation induction distance.

\section{Введение}

Произвольный потенциально взрывоопасный объект (ПВОО) может рассматриваться с позиций системного анализа как сложная система, архитектура которой складывается из некоторых компонентов (подсистем) и из иерархических отношений этих компонентов [1]. В конечном итоге, ПВОО состоит из отдельных элементарных потенциально взрывоопасных объектов (ЭПВОО) [1]. При математическом моделировании производственного ПВОО, в качестве ЭПВОО, моделирующего некоторый реальный объект в составе ПВОО, рассматривается плоский канал или круглая цилиндрическая труба. Например, если в качестве ПВОО рассматривается силосный корпус элеватора, то ЭПВОО представляет собой отдельный силос, который моделируется плоским каналом в случае прямоугольного 
сечения или круглой цилиндрической трубой в случае круглого сечения. Ниже с позиций обеспечения взрывобезопасности рассматриваются только ЭПВОО. Корректная оценка взрывоопасности каждого отдельного ЭПВОО является ключом к оценке взрывоопасности ПВОО в целом [1-3] и предоставляет возможности управления общим уровнем взрывоопасности.

\section{Анализ литературных источников и постановка проблемы}

Проблемы оценки уровня взрывоопасности ЭПВОО и управления (автоматизированного либо автоматического) этим объектом с целью поддержания его во взрывобезопасном состоянии рассмотрены в ряде работ [4-9]. Ни в одной из этих работ не ставился, однако, вопрос об оптимальном управлении взрывоопасностью, что вполне естественно, так как изначально требовалось решить проблему управления взрывоопасностью ЭПВОО в целом, после чего только можно рассматривать и поставить - с той или иной точки зрения - задачу оптимизации.

Критерии оптимальности при управлении ЭПВОО (и вообе ПВОО) для поддержания его во взрывобезопасном состоянии могут быть различными. Наиболее естественными представляются три возможности:

1. Минимизация времени перевода ЭПВОО из относительно взрывоопасного состояния в относительно взрывобезопасное. Эта задача в общем виде не может быть не только решена, но даже поставлена, так как ее постановка и решение существенным образом зависят как от характера конкретного производственного процесса, так и от имеющихся в распоряжении средств предотвращения и подавления взрыва. Хотя, естественно, для конкретного производства постановка и решение подобной задачи не только возможны, но и необходимы.

2. Минимизация расходов по переводу ЭПВОО из одного состояния в другое, более безопасное. Такая постановка задачи возможна, когда: а) заданы начальное (более взрывоопасное) и конечное (менее взрывоопасное) состояния объекта, а переходы из одного состояния в другое могут осуществляться по разным технологическим цепочкам; б) из заданного состояния объекта можно перевести в несколько других, эквивалентных с технологической точки зрения и достаточно безопасных состояний, но затраты на переходы в эти состояния различны.

3. Минимизация уровня взрывоопасности, то есть перевод объекта из данного состояния в наиболее взрывобезопасное из всех допустимых с точки зрения технологического регламента состояний.

\section{Цель и задачи исследования}

Целью настоящего исследования является постановка и решение (в достаточно общем виде) третьей задачи из вышеперечисленных. Причем решение этой задачи может предоставить исходные данные для задачи $2.6-$ имеет смысл минимизировать расходы по переводу ЭПВОО из данного состояния в наиболее взрывобезопасное состояние из всех технологически возможных.

\section{Методы и материалы исследований}

Любой ЭПВОО характеризуется физико-химическими свойствами (динамические свойства) и геометрией своих границ (стенок) (статические свойства). Именно вид геометрии границ позволяет (как это сделано выше) определить и одновременно классифицировать элементарные потенциально взрывоопасные объекты.

Для ЭПВОО оценка взрывоопасности, может быть выражена нечеткой логической переменной $\tilde{E}$, которая определяется соотношением

$$
\tilde{E}=\tilde{F} \wedge \tilde{E}_{R}
$$

где $\tilde{F}$ - нечеткая логическая переменная, выражающая пожароопасность ЭПВОО, $\tilde{E}_{R}-$ нечеткая логическая переменная, выражающая относительную взрывоопасность ЭПВОО. Имеют место неравенства $0 \leq \tilde{\boldsymbol{E}} \leq \mathbf{1}$ ( $\tilde{\boldsymbol{E}}=\mathbf{0}$ означает, что ситуация абсолютно взрывобезопасная, то есть взрыв ни при каких обстоятельствах невозможен; $\tilde{E}=1$ означает, что взрыв неизбежен), $0 \leq \tilde{F} \leq 1$ ( $\tilde{F}=0$ означает, что ситуация абсолютно пожаробезопасная, то есть пожар невозможен; $\tilde{F}=1$ означает, что пожар неизбежен), $0 \leq \tilde{\boldsymbol{E}}_{R} \leq 1 \quad$ ( $\tilde{\boldsymbol{E}}_{R}=$ О означает, что ситуация относительно взрывобезопасная, то есть возникший пожар ни при каких обстоятельствах не перейдет во взрыв; $\tilde{E}_{R}=1$ означает, что при уже возникшем пожаре взрыв неизбежен).

В ряде случаев есть смысл отделить собственно проблему взрывоопасности от проблемы пожароопасности [2]. Очевидно, что чем ниже уровень пожароопасности объекта, тем ниже и уровень его взрывоопасности, что находит прямое отражение в формуле $(1)$, однако величины $\tilde{F}_{\text {и }} \tilde{E}_{R}$ в этой формуле фактически не связаны между собой какой-либо зависимостью. Под относительной взрывоопасностью $\tilde{E}_{R}$ понимается не возможность возникновения пожара (возгорания) с последующим переходом во взрыв, а возможность перехода пожара во взрыв при уже возникшем пожаре. В дальнейшем задача поддержания взрывобезопасности ЭПВОО понимается в смысле обеспечения низкой относительной взрывоопасности объекта, то есть ЭПВОО предполагается (относительно) взрывобезопасным, если $\tilde{E}_{R} \approx 0\left(0 \leq \tilde{E}_{R} \square 1\right)$. Это имеет смысл, так как современные АСУ потенциально взрывоопасными объектами (которые являются также и пожароопасными) в достаточной степени обеспечивают их пожаробезопасность. 
Общая формула для нечеткой величины $\tilde{E}_{R}$ имеет вид

$$
\tilde{E}_{R}=\wedge_{i=1}^{n} \tilde{F}_{i}^{E R}
$$

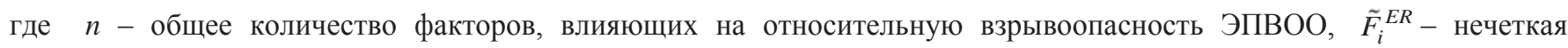
логическая переменная, выражающая влияние $і$-го фактора на относительную взрывоопасность объекта.

Предположим, что рассматриваемый ЭПВОО обладает протяженностью $L$ ( $L$ есть длина трубы или канала), а оценка длины преддетонационного участка составляет $L_{p d i}[10,11]$, тогда можно ввести в рассмотрение нечеткую логическую переменную $\tilde{\boldsymbol{L}}$ с функцией принадлежности $\mu_{L}$, имеющей кусочно-линейный вид, заданный формулами

$$
\mu_{L}=\left\{\begin{array}{l}
\frac{L}{L_{p d i}}, 0 \leq L \leq L_{p d i} \\
1, L_{p d i} \leq L
\end{array}\right.
$$

Как правило, оценка относительной взрывоопасности $\tilde{E}_{R}$ фактически совпадает со значением логической переменной $\tilde{\boldsymbol{L}}$, выражающей соблюдение или несоблюдение длины преддетонационного участка, то есть $\tilde{E}_{R}=\tilde{L}$ и $\tilde{E}=\tilde{F} \wedge \tilde{L}$. Таким образом, если $\tilde{F}=1$, то есть в случае, когда $\tilde{F}$ есть «четкая» истина, $\tilde{E}=\tilde{E}_{R}=\tilde{L}$. Очевидно, что, если $\tilde{E}_{R}=\tilde{L}$, то в случае $L_{p d i} \leq L$ взрыв при возгорании неизбежен: пламя успевает «разогнаться» и создает перед собой ударную волну высокой интенсивности, которая при определенных условиях может стать детонационной волной $[10,11]$. Не исключены и более сложные - по сравнению с формулой $\tilde{E}_{R}=\tilde{L}-$ оценки для нечеткой величины $\tilde{E}_{R}$. Например, если стабилизация пламени наступает при относительно небольших значениях числа Маха [12], есть смысл ввести в рассмотрение некоторую нечеткую переменную, выражающую возможность превышения критического числа Маха (за которым горение переходит во взрыв), а затем включить эту логическую переменную в правую часть соотношения $\tilde{E}_{R}=\tilde{L}$ в виде нечеткой конъюнкции с $\tilde{\boldsymbol{L}}$. При этом формула (1) остается в силе, то есть нечеткая оценка взрывоопасности некоторого ЭПВОО выражается конъюнкцией нечетких оценок пожароопасности и относительной взрывоопасности этого ЭПВОО.

Очевидно, что значение нечеткой переменной $\tilde{L}$ определяется заданной величиной $L$ и расчетной величиной $L_{p d i}$. Расчет $L_{p d i}$ производится по формуле [11]

$$
L_{p d i}=-\frac{4 z_{1} L_{f}}{\left(1-M_{1}\right) M_{1} z_{0}^{2} \beta_{f}\left(\gamma_{1}+1\right)},
$$

где величина $\beta_{f}$ представляет собой некоторую безразмерную константу, близкую к единице $\left(0 \square \beta_{f} \leq 1\right.$, причем, как правило, принимают $\left.\beta_{f}=0,9\right), M_{1}=u_{1} / a_{1}$ - число Маха ( $a_{1}$ - скорость звука в исходной горючей смеси, а $u_{1}-$ скорость нормального горения), $\gamma_{1}$ - отношение теплоемкостей в горючей смеси, $L_{f}-$ протяженность зоны (ламинарного) пламени. Величины $z_{0}$ и $z_{1}$ определяются формулами $[13,14]$

$$
\begin{gathered}
z_{0}=\frac{\delta_{2}}{\delta_{2}+1}\left(-1+\sqrt{\delta_{2}+1-\frac{1}{\delta_{2}}}\right), \\
-\left(1+\frac{\delta_{2}}{\delta_{2}+1} z_{0}\right) z_{1}=z_{0}\left[\frac{1}{2}\left(1+\frac{z_{0}}{2}\right)\left(2 \delta_{2}+1\right)+\left(\frac{\delta_{2}}{\delta_{3}}-1\right)+\delta_{2}^{m}\left(\frac{\delta_{2}+1}{\delta_{2}} z_{0}+\delta_{3}+3\right)\right]+ \\
+\frac{1}{2}\left(\delta_{2}-1\right)\left(1+2 z_{0}+\frac{z_{0}^{2}}{\delta_{3}}\right)
\end{gathered}
$$

где

$$
\delta_{2}=\frac{\rho_{1}}{\rho_{2}}, \delta_{3}=\delta_{2}-\frac{\delta_{2}-1}{e}
$$

$\rho_{1}, \rho_{2}$ - плотности исходной горючей смеси и продуктов сгорания соответственно, а $m(0,5 \leq m \leq 1)$ есть константа, которая в случае гомогенной горючей среды (газовой смеси) представляет собой показатель степени в зависимости динамического коэффициента вязкости газа от температуры. 
Технологический процесс налагает определенные ограничения на физико-химические параметры, определяющие состояние ЭПВОО. То есть давление, температура, плотность (эти три величины не являются независимыми, а связаны уравнением состояния среды), концентрация горючего (в частности, концентрация пыли во взрывоопасной пылевоздушной среде), влажность и другие параметры исходной горючей смеси (гомогенной или гетерогенной) изменяются только в определенных интервалах. В общем случае, эти ограничения определяют также границы изменения каждого из влияющих на относительную взрывоопасность ЭПВОО факторов $\tilde{F}_{i}^{E R}(i=1, \ldots, n)$ в формуле $(2)$. В частном случае, когда $\tilde{E}_{R}=\tilde{L}$, все ограничения, налагаемые характером производства и технологическим процессом на параметры исходной горючей смеси, так или иначе сводятся к ограничениям в виде неравенств для двух величин из правой части формулы (4) с учетом соотношений (5)-(7): $\delta_{2}=\rho_{1} / \rho_{2}-$ коэффициента расширения продуктов сгорания ( $\delta_{2}>1$, так как плотность исходной горючей смеси $\rho_{1}$ всегда больше плотности раскаленных продуктов сгорания $\left.\rho_{2}\right)$ и протяженности зоны пламени $L_{f}$.

Оптимальным с точки зрения взрывобезопасности следует считать такое состояние объекта, для которого нечеткая величина $\tilde{E}_{R}$, выражающая относительную взрывоопасность, минимальна. Таким образом, задача оптимального управления ЭПВОО сводится к поиску минимума функции (2) для $\tilde{E}_{R}$, то есть $\tilde{E}_{R}=\underset{i=1}{n} \tilde{F}_{i}^{E R} \rightarrow \min$ при заданных ограничениях.

В частном случае $\tilde{E}_{R}=\tilde{L}$ оптимальное управление ЭПВОО предполагает минимизацию нечеткой величины $\tilde{L}$ или, что то же, максимизацию функции двух переменных (4) для длины преддетонационного участка $L_{p d i}$ при заданных ограничениях, наложенных на переменные $\delta_{2}$ и $L_{f}$, от которых зависит $L_{p d i}$ (величина $M_{1}$ в правой части (4) не является независимой переменной, так как легко определяется по значениям величины $\delta_{2}$ и скорость нормального горения $u_{1}$ ). Таким образом, задача оптимального управления в данном случае формулируется следующим образом:

$$
\begin{aligned}
L_{p d i}\left(\delta_{2}, L_{f}\right)=- & \frac{4 z_{1} L_{f}}{\left(1-M_{1}\right) M_{1} z_{0}^{2} \beta_{f}\left(\gamma_{1}+1\right)} \rightarrow \max \\
& \left\{\begin{array}{l}
\delta_{2^{*}} \leq \delta_{2} \leq \delta_{2}^{*} \\
L_{f *} \leq L_{f} \leq L_{f}
\end{array}\right.
\end{aligned}
$$

Величины $\delta_{2^{*}}, \delta_{2}^{*}$, представляющие собой соответственно нижнюю и верхнюю границы возможного изменения величины $\delta_{2}$, легко определяются из ограничений, накладываемых на физические параметры технологическим процессом, при помощи законов сохранения (при известной величине $u_{1}$ ). Величины $L_{f}{ }^{*}, L_{f}{ }^{*}$, представляющие собой соответственно нижнюю и верхнюю границы возможного изменения протяженности зоны пламени $L_{f}$, определяются из тех же технологических ограничений при помощи математической теории стационарного горения (случаи гомогенного и гетерогенного горения - принципиально различны).

Учитывая относительную простоту целевой функции в (8), задача максимизации этой функции решается численно для прямоугольной области (9) методом перебора по сетке (равномерного поиска), то есть, по сути, с помощью простого табулирования этой функции.

Для более общего случая целевой функции (2) задачу минимизации следует решать отдельно для каждого конкретного ЭПВОО с учетом особенностей производственного процесса.

\section{Результаты исследований}

Задача оптимального управления (8)-(9) решена для конкретного ЭПВОО, в качестве которого выбран отдельно взятый силос (в реальности такой силос может быть как отдельно стоящим, так и входить в состав силосного корпуса). Проблема взрывобезопасности силосов представляется актуальной, так как по статистике именно на силосы и бункеры приходится почти половина от общего числа взрывов на предприятиях по хранению и переработке зерна, то есть именно силосы представляют собой наиболее взрывоопасные ЭПВОО в таких ПВОО, которыми являются зерновые и комбикормовые производства и хранилища. При загрузке и гравитационной разгрузке силосов в их свободных объемах образуется пылевоздушная смесь (ПВС), способная воспламеняться и гореть. На стенках бункеров и силосов может оседать и накапливаться в значительных количествах мелкодисперсная сухая пыль, которая при внешних возмущениях быстро переходит во взвешенное состояние, также создавая взрывоопасную ПВС. Взрыв в силосе приводит к тяжелым последствиям, так как при этом часто разрушаются боковые стенки и перекрытия, а также деформируется и разрывается выпускной конус под действием давления взрыва. 
При решении задачи оптимального управления (8)-(9) рассматриваются силосы как железобетонные (монолитные и сборные), так и металлические, но обязательно с круглой или прямоугольной (в частности - квадратной) формой поперечного сечения.

Для оптимального управления уровнем взрывоопасности отдельно взятого силоса в среде программирования Visual Basic разработана вычислительная программа «Оптимальное управление взрывоопасностью отдельных силосов» («SilosOtdelniyOpt»), которая включена в состав программного комплекса «Оценка взрывоопасности отдельных силосов» («SilosOtdelniy»), разработанного ранее [15]. Интерфейс программы предоставляет пользователю возможность выбрать один из стандартных железобетонных силосов или же самостоятельно задать форму и размеры силоса (железобетонного или металлического). Дальнейшие расчеты требуют задания вида ПВС (зернового продукта), концентрации пыли, влажности, температуры и дисперсности (среднего размера пылевых частиц). Все эти величины могут измеряться при помощи стандартных метрологических приборов в режиме эксплуатации зернохранилища или зерноперерабатывающего предприятия (а сама программа в этом случае является составной частью программного обеспечения соответствующей автоматизированной системы управления). В результате расчетов пользователь (как правило, им является оператор-технолог) получает информацию:

1) об уровне взрывоопасности силоса, о длине преддетонационного участка и о времени возможного перехода медленного горения (пожара) во взрыв;

2) о наиболее взрывобезопасном состоянии силоса из всех его возможных (в рамках технологического процесса) состояний.

Эта информация является необходимой для принятия обоснованного решения по обеспечению взрывобезопасности и/или взрывозащиты силоса.

\section{Обсуждение результатов}

Решение задачи об оптимальном управлении уровнем взрывоопасности отдельно взятого силоса создает предпосылки для решения задачи об оптимальном управлении взрывоопасностью силосного корпуса в целом, а в перспективе - об оптимальном управлении взрывоопасностью всего элеватора или другого зернового предприятия. Другое направление дальнейших исследований связано с рассмотрением ситуаций, когда нечеткая оценка относительной взрывоопасности ЭПВОО не сводится к расчету длины преддетонационного участка $L_{p d i}$. В этом случае существенно усложняется вид целевой функции.

\section{Выводы}

1. Рассмотрен вопрос об оптимальном управлении потенциально взрывоопасным объектом. Показано, что критерии оптимальности могут быть различными. Поставлена задача об оптимальном управлении уровнем взрывоопасности потенциально взрывоопасного объекта.

2. Поставленная задача решена в общем виде для простейшего случая, когда оценка уровня относительной взрывоопасности элементарного потенциально взрывоопасного объекта сводится к расчету длины преддетонационного участка. Решение реализовано в виде программного продукта.

3. Реализована программа оптимального управления уровнем относительной взрывоопасности отдельно взятого силоса. Данная программа может стать частью программного обеспечения АСУ предприятия по хранению и переработке зерна.

\section{Список использованных источников}

[1] Волков В.Э. Информационная модель потенциально взрывоопасного объекта. Часть 1. // Автоматизация технологических и бизнес-процессов, 2012. - №№9,10. - С. 3-11.

[2] Волков В.Э. Информационная модель потенциально взрывоопасного объекта. Часть 2 // Автоматизация технологических и бизнес-процессов, 2012. - №№11,12. - С. 3-9.

[3] Волков В.Э. Информационная модель потенциально взрывоопасного объекта. Часть 3 // Автоматизация технологических и бизнес-процессов, 2013. - №№13,14. - С. 3-9.

[4] Volkov V.E. Decision Support Systems on Hazards of Industrial Explosions // Seventh International Symposium on Hazards, Prevention and Mitigation of Industrial Explosions: Thirteeenth International Colloquium on Dust Explosions \& Eighth Colloquim on Gas, Vapor, Liquid, and Hybrid Explosions. St. Petersburg, Russia. July 7-11, 2008. - St. Petersburg, 2008. - Vol.3. - P.343-347.

[5] Волков В.Э. Управление процессом перехода медленного горения во взрыв // Автоматизация технологических и бизнес-процессов, 2010. - №2. - С. 5-13.

[6] Волков В.Э. Специфика и возможности управления взрывоопасными объектами // Автоматизация технологических и бизнес-процессов, 2014. - №18. - С. 4-12.

[7] Попов А.С. Нечеткие оценки взрывоопасности объектов зерноперерабатывающего предприятия / А.С. Попов, В.Э. Волков // Wschodnioeuropejskie Czasopismo Naukowe. - Warszawa 2016. - № 5(9), część 3. - S. 11-15.

[8] Волков В.Э. Математическое обеспечение современных АСУ потенциально взрывоопасными объектами / В.Э.Волков, Н.А.Макоед // Сборник научных трудов международной научной конференции 
«Интеллектуальные системы принятия решений и проблемы вычислительного интеллекта (ISDMCI-2017)», 21-27 мая 2018 г., Железный Порт, Украина. - Херсон: Видавництво ПП Вишемирський В.С., 2018. - С.42-44.

[9] Волков В.Э. Теория принятия решений как основа управления сложными системами / В.Э.Волков, Н.А.Макоед // Автоматизация технологических и бизнес-процессов, 2010. - №1 . - С. 6-10.

[10] Волков В.Э. Розрахунок довжини преддетонаційної ділянки //Наукові праці Одеської національної академії харчових технологій. - Одеса, 2009. - Вип. 36. Т.1. - С. 285-288.

[11] Volkov V.E. Deflagration-to-detonation transition and the detonation induction distance estimation // Праці Одеського політехнічного університету. - Одеса, 2014. - Вип. 1(43) - С. 120-126.

[12] Волков В.Э. Неустойчивость пламени в идеальной сжимаемой среде и переход медленного горения во взрыв // Наукові праці Одеської національної академії харчових технологій. - Одеса, 2010. - Вип. 38. Т.1. - С. 325332.

[13] Aslanov, S. On the instability and cell structure of flames / S. Aslanov, V. Volkov // Archivum combustionis. - 1992. - Vol. 12, Nr. 1-4. - P. 81-90.

[14] Aslanov, S.K. Instability and Structure of Flames / S.K. Aslanov, V.E. Volkov. In: Pulsed and Continuous Detonations. - Moscow: TORUS PRESS, 2006. - P.28-31.

[15] Волков В.Э. Программа оценки взрывоопасности силоса // Наукові праці Одеської національної академії харчових технологій. - Одеса, 2014. - Вип. 46. Т.1. - С. 229-235.

\section{References}

[1] Volkov V.E. Informatsionnaya model' potentsial'no vzryvoopasnogo ob"yekta. Chast' 1 // Avtomatizatsiya tekhnologicheskikh i biznes-protsessov, 2012. - №№9,10. - S. 3-11.

[2] Volkov V.E. Informatsionnaya model' potentsial'no vzryvoopasnogo ob"yekta. Chast' 2 // Avtomatizatsiya tekhnologicheskikh i biznes-protsessov, 2012. - №o11,12. - S. 3-9.

[3] Volkov V.E. Informatsionnaya model' potentsial'no vzryvoopasnogo ob"yekta. Chast' 2 // Avtomatizatsiya tekhnologicheskikh i biznes-protsessov, 2013. - №№13,14. - S. 3-9.

[4] Volkov V.E. Decision Support Systems on Hazards of Industrial Explosions // Seventh International Symposium on Hazards, Prevention and Mitigation of Industrial Explosions: Thirteeenth International Colloquium on Dust Explosions \& Eighth Colloquim on Gas, Vapor, Liquid, and Hybrid Explosions. St. Petersburg, Russia. July 7-11, 2008. - St. Petersburg, 2008. - Vol.3. - P.343-347.

[5] Volkov V.E. Upravleniye protsessom perekhoda medlennogo goreniya vo vzryv // Avtomatizatsiya tekhnologicheskikh i biznes-protsessov, 2010. - №2. - S. 5-13.

[6] Volkov V.E. Spetsifika i vozmozhnosti upravleniya vzryvoopasnymi ob"yektami // Avtomatizatsiya tekhnologicheskikh i biznes-protsessov, 2014. - №18. - S. 4-12.

[7] Popov A.S. Nechetkiye otsenki vzryvoopasnosti ob"yektov zernopererabatyvayushchego predpriyatiya / A.S. Popov, V.E. Volkov // Wschodnioeuropejskie Czasopismo Naukowe. - Warszawa 2016. - № 5(9), część 3. - S. 11-15.

[8] Volkov V.E. Matematicheskoye obespecheniye sovremennykh ASU potentsial'no vzryvoopasnymi ob"yektami / V.E.Volkov, N.A.Makoyed // Sbornik nauchnykh trudov mezhdunarodnoy nauchnoy konferentsii «Intellektual'nyye sistemy prinyatiya resheniy i problemy vychislitel'nogo intellekta (ISDMCI-2017)», 21-27 maya 2018 g., Zheleznyy Port, Ukraina. - Kherson: Vidavnitstvo PP Vishemirs'kiy V.S., 2018. - S.42-44.

[9] Volkov V.E. Teoriya prinyatiya resheniy kak osnova upravleniya slozhnymi sistemami / V.E.Volkov, N.A.Makoyed // Avtomatizatsiya tekhnologicheskikh i biznes-protsessov, 2010. - №1 . - S. 6-10.

[10] Volkov V.E. Rosrahunok dovzhini preddetonatsiyno i dilyanki // Naukovi Pratsi Odeskoi Natsial'noi Akademii Khartovykh Tehnologiy. - Odesa, 2009. - Vip. 36. T.1. - S. 285-288.

[11] Volkov V.E. Deflagration-to-detonation transition and the detonation induction distance estimation // Pratsi Odes'kogo Polytechnichnogo Universytetu. - Odesa, 2014. - Vip. 1 (43) - pp. 120-126.

[12] Volkov V.E. Neustoychivost' plameni v ideal'noy szhimayemoy srede i perekhod medlennogo goreniya vo vzryv // Naukovi Pratsi Odeskoi Natsial'noi Akademii Khartovykh Tehnologiy. - Odesa, 2010. - Vip. 38. T.1. - S. 325-332.

[13] Aslanov, S. On the instability and cell structure of flames / S. Aslanov, V. Volkov // Archivum combustionis. - 1992. - Vol. 12, Nr. 1-4. - P. 81-90.

[14] Aslanov, S.K. Instability and Structure of Flames / S.K. Aslanov, V.E. Volkov. In: Pulsed and Continuous Detonations. - Moscow: TORUS PRESS, 2006. - P.28-31.

[15] Volkov V.E. Programma otsenki vzryvoopasnosti silosa // Naukovi Pratsi Odeskoi Natsial'noi Akademii Khartovykh Tehnologiy. - Odesa, 2014. - Vip. 46. T.1. - S. 229-235. 